\title{
Impact of Nutritional Practices on Rural Women through Series of Extension Games
}

\author{
Mamta Tiwari* \\ Director Prioritization, Monitoring, Evaluation Agriculture University, Kota (Rajasthan), India \\ *Corresponding author
}

A B S T R A C T

\begin{tabular}{|l|}
\hline Ke y w or d s \\
Nutritional \\
knowledge, \\
Good health. \\
\hline Article Info \\
\hline $\begin{array}{l}\text { Accepted: } \\
\text { 10 September } 2017 \\
\text { Available Online: } \\
10 \text { October } 2017\end{array}$ \\
\hline
\end{tabular}

Nutritional knowledge for maintaining good health is necessarily required for the upliftment of country. Food products including cereal, pulses, fruits, vegetables, milk etc. contains high amount of protein, vitamins, minerals, calcium, carbohydrates, calories, dietary fibre and fats and have numerous therapeutic benefits. Due to their health beneficial properties they were incorporated with other ingredients or treated with processing technologies and turned into a new product and projected for estimation of moisture, protein, fat, carbohydrate, calcium and iron. It is necessary to have the knowledge of nutrient contain in food and different technologies to enhance nutritional value. Learning through fun is today's need. For imparting any knowledge games can be the effective source. Present study was conducted in the year 2015-16 with the women of Arjunpura and Raipura villages of Kota District of Rajasthan. In the present study series of games on 6 different aspects Like nutrients and its sources, diseases due to nutritional deficiencies, enhancement in nutritional content of food, processing and value addition of food products and adulteration in food products were prepared and introduced to 60 women in 5 groups (12 women in each group). They played in different groups alternatively 3 times each group and their extent of awareness, knowledge, development in skills, adoption, change in behavioural practices influential practices were judged through mean influence by high, moderate and low category. Result showed that nutritional awareness, knowledge, and influential practices towards nutrient content and its enhancement in food were highly increased $(86.66 \%, 81.66 \%, 83.33 \%)$ respectively. It was also found out that skill development, adoption and their behavioural practices were moderately influenced $(30.00 \%, 31.66 \%, 35.00 \%)$ respectively whereas low adoption was found in the area of adoption, influential practices, skill development and behavioural changes $16.66 \%, 13.33 \%, 11.66 \%, 10.00 \%$ ) respectively

\section{Introduction}

It is necessary to have the knowledge of nutrient content in food and different technologies to enhance nutritional value. Learning through fun is today's need. For imparting any knowledge games can be the effective source. Nutritional knowledge for maintaining good health is necessarily required for the upliftment of country. Significantly enhanced the Level of nutritional knowledge among young women (Jain et al., 2013a). Nutrition information of food labels provide information related to 
food ingredient, nutrient contents and preparation methods. Thus all people should be promoted for the habit of reading nutrition label while purchasing any food items (Jain et $a l ., 2013 b)$. These types of information can be imparted through recreation. Food processing and preservation of food items is very essential for development of any new food items. Food products including cereal, pulses, fruits, vegetables, milk etc. contains high amount of protein, vitamins, minerals, calcium, carbohydrates, calories, dietary fibre and fats and have numerous therapeutic benefits. Due to their health beneficial properties they were incorporated with other ingredients or treated with processing technologies and turned into a new product and projected for estimation of moisture, protein, fat, carbohydrate, calcium and iron. Some examples of novel food items are fruit yoghurt (Jain et al., 2013f), instant kheer mix (Gupta et al., 2014), Vegetable cereal mix (Gupta et al., 2016).

Nutrameal- shakti aahar (Tiwari et al., 2017) etc. Area of food and Nutrition is very wide and is applicable in day to day life of individual to uplight their health status. With the slight change in their life they can change their health and nutritional status. Therefore in present work influence of series of extension games was assessed in Nutritional practices of rural women.

\section{Materials and Methods}

Present study was conducted in the year 201516 with the women of Arjunpura and Raipura villages of Kota District of Rajasthan. In the present study series of games on 6 different aspects like Nutrients and its sources, diseases due to nutritional deficiencies, enhancement in nutritional content of food, processing \& value addition of food products and adulteration in food products were prepared and introduced to 60 women in 5groups (12 women in each group). They played in different groups alternatively 3 times each group and their extent of awareness, knowledge, development in skills, adoption, change in behavioural practices influential practices were judged through mean influence by high, moderate and low category.

\section{Results and Discussion}

After playing and practicing the game the nutritional awareness, knowledge, and influential practices towards nutrient content and its enhancement in food, skill development, adoption and the behavioural practices of rural women were found out.

Table.1 Mean influence on nutritional practices of rural women through series of extension games

\begin{tabular}{|l|l|c|c|c|}
\hline S. No. & \multirow{2}{*}{ Aspects of Nutritional Development } & \multicolumn{2}{|c|}{ Mean Influence } \\
\cline { 3 - 4 } & & High & Moderate & Low \\
\hline 1. & Awareness of Nutrients in food & $52(86.66 \%)$ & $6(10.00 \%)$ & $2(3.34 \%)$ \\
\hline 2. & $\begin{array}{l}\text { Knowledge of sources of Nutrients/ deficiency } \\
\text { deceases }\end{array}$ & $49(81.66 \%)$ & $6(10.00 \%)$ & $5(8.34 \%)$ \\
\hline 3. & $\begin{array}{l}\text { Skill Development in enhancing/saving nutrients in } \\
\text { food }\end{array}$ & $35(58.34 \%)$ & $18(30.00 \%)$ & $7(11.66 \%)$ \\
\hline 4. & Adoption through improvement in nutritional habits & $31(51.67 \%)$ & $19(31.66 \%)$ & $10(16.66 \%)$ \\
\hline 5. & $\begin{array}{l}\text { Behaviour Changes in selection and preparation of } \\
\text { food }\end{array}$ & $33(55.00 \%)$ & $21(35.00 \%)$ & $6(10.00 \%)$ \\
\hline 6. & Influential Practices during intake of nutritional food & $50(83.33 \%)$ & $2(3.34 \%)$ & $8(13.33 \%)$ \\
\hline
\end{tabular}


Series of Extension games, Krishi Vigyan Kendra, Kota, Agriculture University, Kota

Sources of Nutrients

\begin{tabular}{|c|c|c|c|c|c|}
\hline Protein & Calcium & Iron & $\begin{array}{l}\text { Rice, } \\
\text { bananas, } \\
\text { cashewrs, } \\
\text { ghee, } \\
\text { grain, } \\
\text { groundnut }\end{array}$ & $\begin{array}{l}\text { Pulses, Egg, } \\
\text { Milik }\end{array}$ & $\begin{array}{l}\text { Lemon, } \\
\text { orange, } \\
\text { tomato, Amla, } \\
\text { green chill, } \\
\text { turnips, } \\
\text { ginger }\end{array}$ \\
\hline Vitamin- 'A $A^{\prime}$ & Vitamin-' $\mathbf{B}$ ' & Vitamin-"C" & $\begin{array}{l}\text { Cucumber, } \\
\text { potato, } \\
\text { raisins }\end{array}$ & $\begin{array}{l}\text { Potato, Sweet } \\
\text { potato, } \\
\text { beetroot }\end{array}$ & $\begin{array}{l}\text { Spinach, } \\
\text { pumplin, } \\
\text { almond, tofu }\end{array}$ \\
\hline $\begin{array}{l}\text { Calorie } \\
\text { Energy }\end{array}$ & $\begin{array}{c}\text { Carbo } \\
\text { hydrates }\end{array}$ & Kalizum & $\begin{array}{l}\text { Fegs, milk, } \\
\text { butter, } \\
\text { fruit, } \\
\text { yellow } \\
\text { carrots }\end{array}$ & $\begin{array}{l}\text { Cabbage, } \\
\text { radish, } \\
\text { garlic, onion }\end{array}$ & $\begin{array}{l}\text { Almond, } \\
\text { cheese, white } \\
\text { sessame, } \\
\text { spinach, millk, } \\
\text { tomato } \\
\text { powder }\end{array}$ \\
\hline Florin & Copper & Vitamin 'E' & $\begin{array}{l}\text { Millk, millk } \\
\text { products, } \\
\text { papayra, } \\
\text { banana. }\end{array}$ & $\begin{array}{l}\text { Dry fruits, } \\
\text { grain, bitter, } \\
\text { gourd, leafy } \\
\text { vegetables, } \\
\text { oilseed }\end{array}$ & $\begin{array}{l}\text { Podina, } \\
\text { turmeric, } \\
\text { pomegranate }\end{array}$ \\
\hline Antibodies & $\frac{\text { Vitamin 'B' }}{12}$ & Riboflevin & $\begin{array}{l}\text { Peanut, } \\
\text { Beans, } \\
\text { peas, } \\
\text { Garlie }\end{array}$ & $\begin{array}{l}\text { Salt, grapes, } \\
\text { dates, } \\
\text { pineapple, } \\
\text { black pepper, } \\
\text { radish }\end{array}$ & $\begin{array}{l}\text { Grain, cheese, } \\
\text { millk \&s millk } \\
\text { products }\end{array}$ \\
\hline $\begin{array}{c}\text { Nicine } \\
\text { Vitamin 'B' } \\
3\end{array}$ & Zink & Sulfer & $\begin{array}{l}\text { Garlic, } \\
\text { honey, } \\
\text { apple, amla, } \\
\text { cabbage }\end{array}$ & $\begin{array}{l}\text { Mushrooms, } \\
\text { linseed, } \\
\text { walnut, } \\
\text { soybean }\end{array}$ & $\begin{array}{l}\text { Radish, } \\
\text { Cabbage, }\end{array}$ \\
\hline Vitamin 'D" & Magnesium & Sodium & $\begin{array}{l}\text { Green leafy } \\
\text { vegetable, } \\
\text { saunf, } \\
\text { spices, } \\
\text { clove, } \\
\text { omum }\end{array}$ & $\begin{array}{l}\text { Coffee, } \\
\text { peanut, peas }\end{array}$ & $\begin{array}{l}\text { Banana, } \\
\text { dry fruits, } \\
\text { mustard, } \\
\text { cocoa, } \\
\text { roasted } \\
\text { seasame } \\
\text { seeds }\end{array}$ \\
\hline $\begin{array}{l}\text { Vitamin } \\
\text { ' } \mathbf{K} \text { ' }\end{array}$ & Cadmium & Iodine & $\begin{array}{l}\text { Sunshin, } \\
\text { mink, } \\
\text { whole } \\
\text { grains }\end{array}$ & $\begin{array}{l}\text { Barley } \\
\text { sesame, } \\
\text { pulses, } \\
\text { cocoa } \\
\text { powder }\end{array}$ & $\begin{array}{l}\text { Rice, grain, } \\
\text { banana }\end{array}$ \\
\hline
\end{tabular}

Processed Food Products

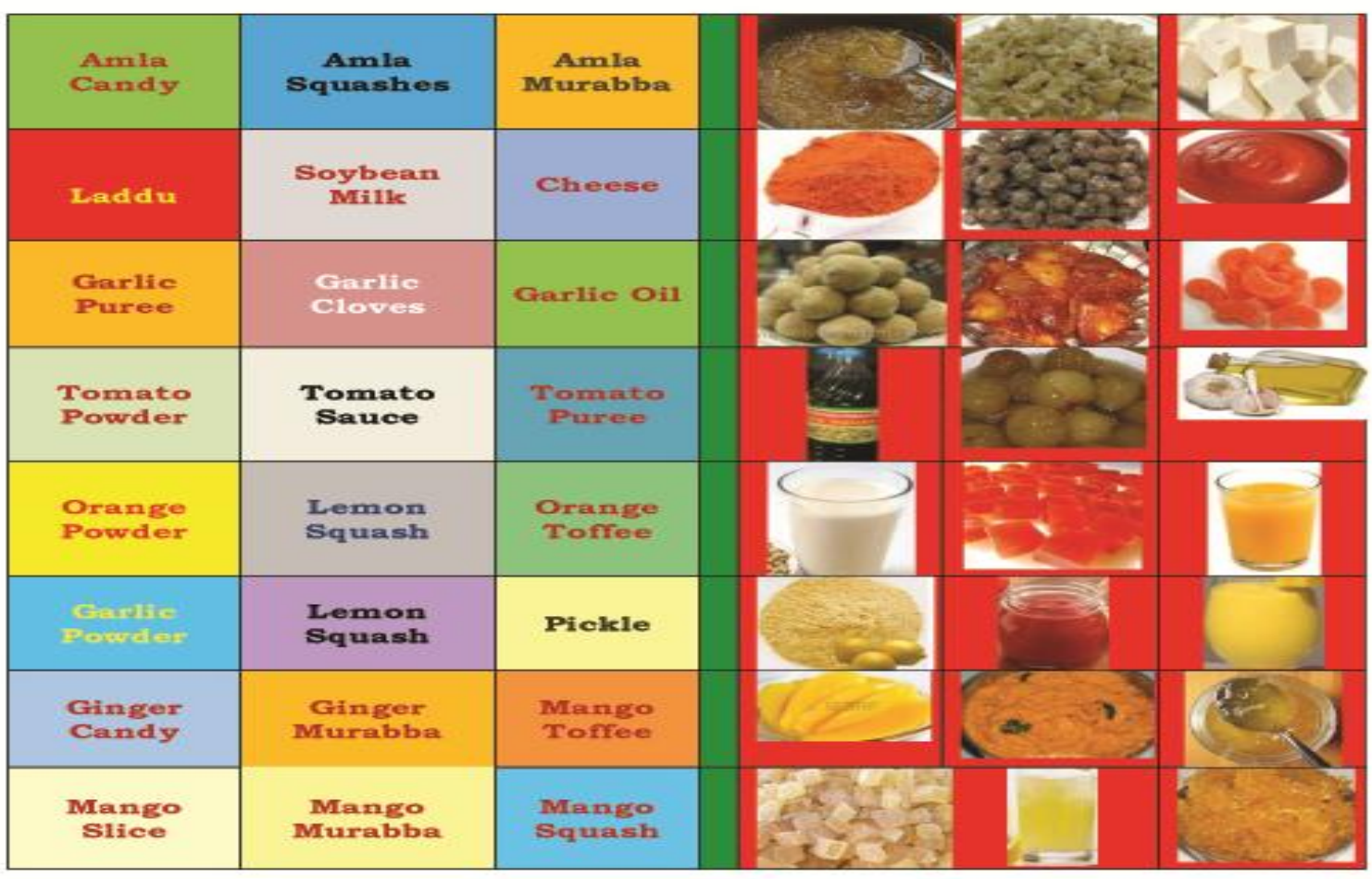




\section{Enhancement of Nutritional Content of Food}

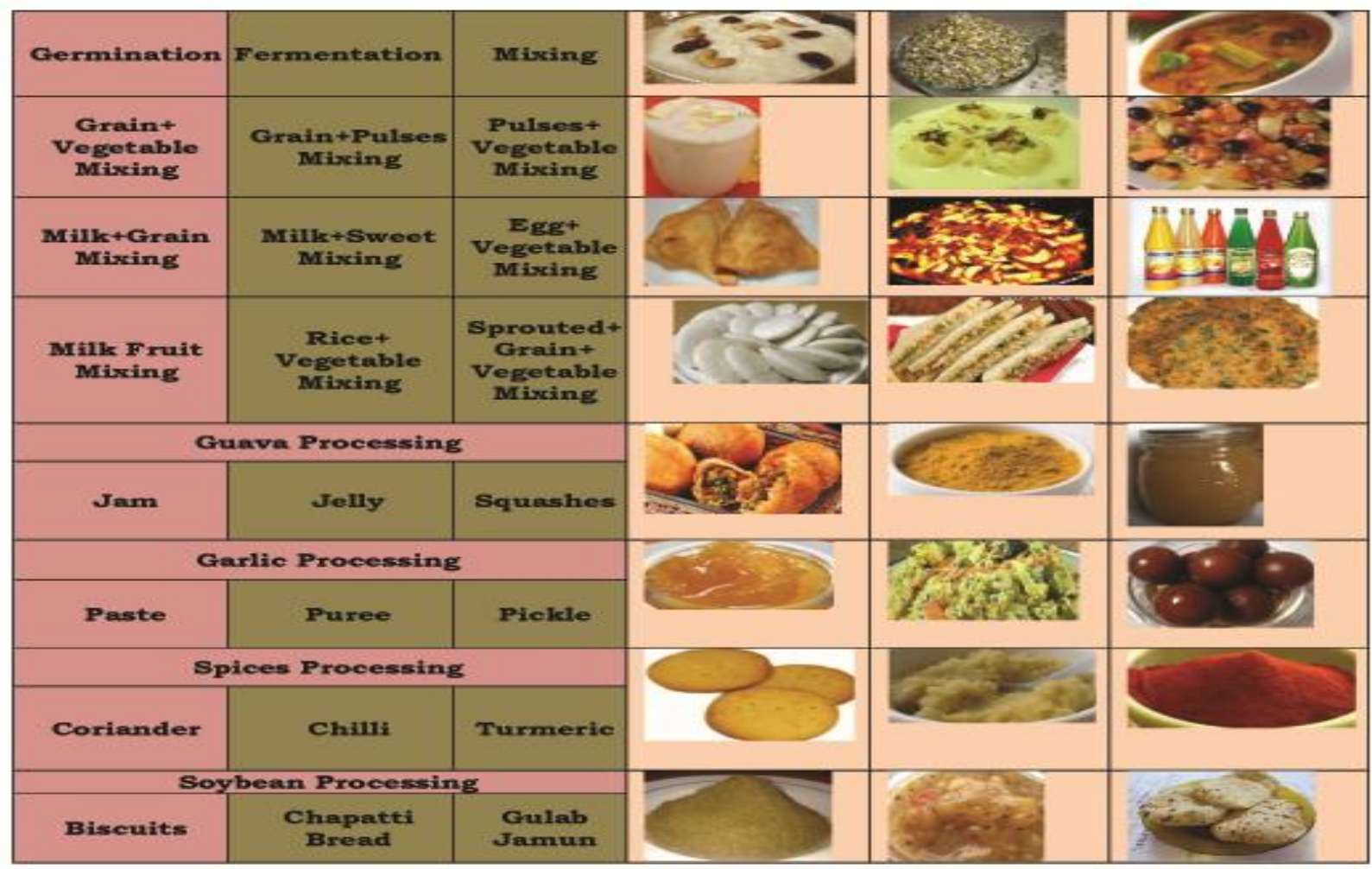

Deficiency Diseases

\begin{tabular}{|c|c|c|c|c|c|}
\hline $\begin{array}{l}\text { Vienmin iA" } \\
\text { Deficiency }\end{array}$ & $\begin{array}{c}\text { Nicine } \\
\text { Deficiency }\end{array}$ & $\begin{array}{l}\text { Ribo Mlavin } \\
\text { Deficiency }\end{array}$ & $\begin{array}{l}\text { Heart } \\
\text { disease }\end{array}$ & $\begin{array}{l}\text { Night } \\
\text { blindiness }\end{array}$ & Scurvy \\
\hline $\begin{array}{c}\text { Calcium } \\
\text { Deficiemey }\end{array}$ & $\begin{array}{c}\text { Energy } \\
\text { Deficieney }\end{array}$ & $\begin{array}{l}\text { Vitamin 'C' } \\
\text { Deficiency }\end{array}$ & Goiter & $\begin{array}{l}\text { Malmutrition } \\
\text { (Jworashiorikor) }\end{array}$ & Riokets \\
\hline Fat Erxcess & $\begin{array}{c}\text { Protein } \\
\text { Deficiency }\end{array}$ & $\begin{array}{l}\text { Antibodies } \\
\text { Deficieney }\end{array}$ & Osteoporosis & Florosis & Cholities \\
\hline $\begin{array}{l}\text { Iodine } \\
\text { Deficieney }\end{array}$ & $\begin{array}{c}\text { Irom } \\
\text { Deficieney }\end{array}$ & $\begin{array}{l}\text { Vitamin } \\
\text { Deficiency }\end{array}$ & Pellagra & Slkin disease & Allergy \\
\hline $\begin{array}{c}\text { Copper } \\
\text { Defieteney }\end{array}$ & $\begin{array}{l}\text { Thiamin } \\
\text { Deficiency }\end{array}$ & $\begin{array}{l}\text { Magnesium } \\
\text { Deficiency }\end{array}$ & $\begin{array}{l}\text { Malmatrition } \\
\text { (Marasmus) }\end{array}$ & A blood olot & Beri-Beri \\
\hline $\begin{array}{l}\text { Kalium } \\
\text { Deficiomey }\end{array}$ & $\begin{array}{l}\text { Sulfux } \\
\text { Doficioney }\end{array}$ & $\begin{array}{l}\text { Florin } \\
\text { Excoess }\end{array}$ & $\begin{array}{l}\text { Nervous } \\
\text { disorder }\end{array}$ & Anemia & Impotency \\
\hline $\begin{array}{l}\text { Tension, } \\
\text { Smoking } \\
\text { Exceess }\end{array}$ & $\begin{array}{c}\text { Zink } \\
\text { Exceess }\end{array}$ & $\begin{array}{l}\text { Vitamin ' } \mathbf{K} \text { ' } \\
\text { Deficiency }\end{array}$ & $\begin{array}{c}\text { Blood } \\
\text { pressure }\end{array}$ & $\begin{array}{l}\text { Paralysis } f \\
\text { Muscular } \\
\text { wealeness }\end{array}$ & $\begin{array}{c}\text { Mental } \\
\text { imbalance }\end{array}$ \\
\hline $\begin{array}{l}\text { Sodium } \\
\text { Deficiency }\end{array}$ & $\begin{array}{l}\text { Exergy } \\
\text { Exceess }\end{array}$ & $\begin{array}{l}\text { Witamin 'E' } \\
\text { Deficiency' }\end{array}$ & Peptic ulcex & $\begin{array}{c}\text { Somi } \\
\text { physical } \\
\text { developmemt }\end{array}$ & Obesity \\
\hline
\end{tabular}


Adulteration in food products

\begin{tabular}{|c|c|c|c|c|c|}
\hline Salt & Red Chilli & Tea leaves & $\begin{array}{c}\text { Charcoal } \\
\text { Grass } \\
\text { Seed }\end{array}$ & $\begin{array}{l}\text { White } \\
\text { Stome } \\
\text { Sawdust }\end{array}$ & $\begin{array}{l}\text { Chicken } \\
\text { Seed }\end{array}$ \\
\hline $\begin{array}{l}\text { Milk } \\
\text { Slim }\end{array}$ & Pure Ghee & Coffee & Ararot & $\begin{array}{l}\text { Papaya } \\
\text { Seeds }\end{array}$ & Cheap Oil \\
\hline Cumin & $\begin{array}{l}\text { Black } \\
\text { Pepper }\end{array}$ & $\begin{array}{l}\text { Pies } \\
\text { Spices }\end{array}$ & Water & $\begin{array}{l}\text { Kesari } \\
\text { Pulse }\end{array}$ & $\begin{array}{l}\text { Sugax } \\
\text { Syrup }\end{array}$ \\
\hline $\begin{array}{l}\text { Milk } \\
\text { Thick }\end{array}$ & Alcohol & $\begin{array}{c}\text { Vegetable } \\
\text { Ghee }\end{array}$ & $\begin{array}{l}\text { Brick } \\
\text { Color }\end{array}$ & $\begin{array}{l}\text { Wood } \\
\text { Powder }\end{array}$ & $\begin{array}{l}\text { Husk } \\
\text { Powdex }\end{array}$ \\
\hline Saffron & $\begin{array}{l}\text { Cloves } \\
\text { Cardamom }\end{array}$ & Homey & $\begin{array}{c}\text { Vegetable } \\
\text { Ghee }\end{array}$ & $\begin{array}{c}\text { Challe } \\
\text { gum } \\
\text { Starch }\end{array}$ & Oil Pulling \\
\hline Jaggery & Coriander & Pulse & $\begin{array}{c}\text { Jute } \\
\text { Clipping }\end{array}$ & $\begin{array}{l}\text { Methanol } \\
\text { poisonous } \\
\text { liquox }\end{array}$ & $\begin{array}{c}\text { Use of } \\
\text { cadmium } \\
\text { characters }\end{array}$ \\
\hline $\begin{array}{l}\text { Wheat } \\
\text { Floux }\end{array}$ & Apple & Asafoetida & $\begin{array}{c}\text { Led } \\
\text { chromate }\end{array}$ & $\begin{array}{l}\text { Metenyl } \\
\text { yellow } \\
\text { collox }\end{array}$ & $\begin{array}{c}\text { Lead } \\
\text { arsenate }\end{array}$ \\
\hline Turmeric & $\begin{array}{l}\text { Silver } \\
\text { Lining }\end{array}$ & $\begin{array}{l}\text { Fruit } \\
\text { Juice }\end{array}$ & $\begin{array}{l}\text { Sand } \\
\text { Chalk } \\
\text { Powdex }\end{array}$ & Cow-dung & Lead Work \\
\hline
\end{tabular}

Answer Sheet

Game Series 1 to 5

\begin{tabular}{|l|l|l|l|l|l|}
\hline & \multicolumn{4}{l}{ Question Code } & \multicolumn{3}{l}{ Answer Code } \\
\hline 1 & 2 & 3 & 7 & 1 & 6 \\
\hline 4 & 5 & 6 & 10 & 8 & 12 \\
\hline 7 & 8 & 9 & 4 & 18 & 15 \\
\hline 10 & 11 & 12 & 2 & 3 & 9 \\
\hline 13 & 14 & 15 & 5 & 21 & 14 \\
\hline 16 & 17 & 18 & 13 & 11 & 24 \\
\hline 22 & 20 & 21 & 22 & 16 & 20 \\
\hline
\end{tabular}

Table 1 revealed that nutritional Awareness of Nutrients in food, knowledge of sources of Nutrients/ deficiency deceases, and Influential
Practices during intake of nutritional food towards nutrient content and its enhancement in food were highly increased $(86.66 \%$, 
$81.66 \%, 83.33 \%)$ respectively. It was also found out that skill development, adoption and their behavioural Changes in selection and preparation of food were moderately influenced $(30.00 \%, 31.66 \%, 35.00 \%)$ respectively whereas low adoption was found in the area of adoption, influential practices, Skill Development in enhancing/saving nutrients in food and behavioural changes $16.66 \%, \quad 13.33 \%, \quad 11.66 \%, \quad 10.00 \%$ ) respectively

It can be concluded that knowledge regarding nutrient content and nutritional practices can be easily imparted in rural/semiliterate individual through fun/recreational activities. These types of nutritional recreational activities will not only help to ease their stress but also will help them to learn and adopt right nutritional practices in a fun manner. The effects of these activities will be long lasting and will help the individuals to improve their health and nutritional status through their indulgence towards healthy and nutritional practices.

\section{References}

Gupta, K., Srivastava S, Jain P and Jain M. Process optimization for formulating Trigonella foenum-graecum and Gymnema sylvestre added vegetable cereal mix using response surface methodology. Journal of Nutritional
Health and Food Engineering. 2016; 4(3):00129.

Gupta, K., Verma M, Jain P and Jain M. Process optimization for producing cowpea added instant kheer mix using response surface methodology. Journal of Nutritional Health and Food Engineering. 2014; 1(5):1-9.

Jain, M., Gupta K and Jain P. A pilot study on the impact of nutrition education on knowledge and practices pertaining to anaemia in young women. Inventi Impact: Life Style. 2013a; (3):107-112.

Jain, M., Gupta K, Goyal A and Jain P. Development and appraisal of nutritional, sensory and keeping quality of various form of papaya yoghurt. Beverages and Food World. 2013c; 40(9):32-34.

Jain, M., Jain P, Gupta K and Sharma S. Understanding and use of nutrition information on food labels by consumers. IRC'S International Journal of Multidiciplinary Research in Social and Management Sciences. 2013b; $1(4): 42-47$.

Tiwari, M., Gupta K, Sanadya G. Fortified Protein rich Soya Product "Nutrameal Shakti Aahar" and its Sensory, Nutritional and Microbiological Analysis. Asian Journal of Home Science. 2017; 12(2). (Accepted for publication)

\section{How to cite this article:}

Mamta Tiwari. 2017. Impact of Nutritional Practices on Rural Women through Series of Extension Games. Int.J.Curr.Microbiol.App.Sci. 6(10): 1069-1074.

doi: https://doi.org/10.20546/ijcmas.2017.610.129 